\title{
Streamer dynamics in gases containing dust particles
}

\author{
Natalia Yu Babaeva ${ }^{1}$, Ananth N Bhoj ${ }^{2}$ and Mark J Kushner ${ }^{1,3}$ \\ ${ }^{1}$ Department of Electrical and Computer Engineering, Iowa State University, Ames, \\ IA 50011, USA \\ ${ }^{2}$ Department of Chemical and Biomolecular Engineering, University of Illinois, \\ 1406 W Green St., Urbana, IL 61801, USA \\ E-mail:mjk@iastate.edu
}

Received 8 January 2006, in final form 30 May 2006

Published 5 July 2006

Online at stacks.iop.org/PSST/15/591

\begin{abstract}
Atmospheric pressure plasmas, and streamers in particular, sustained in air often encounter dust or aerosol particles having sizes of a few to tens of micrometres. The dynamics of streamers intersecting such particles are of interest due to their possible use for functionalizing the surfaces of the particles. Using a 2-dimensional plasma hydrodynamics model having an unstructured mesh, the consequences of dust particles on streamer dynamics were investigated while varying the particle size, shape and material properties. We found that while small dielectric particles $(<$ tens of micrometres) are enveloped by the streamer, larger particles can intercept and reinitiate streamers. By increasing the permittivity and capacitance of the particles, streamer interception and re-initiation may also occur on smaller particles. The presence of multiple particles in the path of a streamer can increase the speed of the avalanche front due to synergistic polarization of the particles.
\end{abstract}

\section{Introduction}

The propagation of streamers in atmospheric pressure electrical discharges is of interest in plasma remediation of toxic gases, ozone production and functionalization of surfaces [1]. The air (or other gases) in these non-pristine environments is often contaminated with particles or aerosols having sizes of tens to hundreds of micrometres. These particles can be generated within the plasma by nucleation, injected externally into the plasma or occur naturally (such as aerosols [2]). The particles may have varying degrees of conductivity, from metallic to classically dielectric.

The physics of dusty, low pressure plasmas has attracted considerable attention from the viewpoint of both the basic plasma physics of non-ideal systems and from the perspective of plasma applications such as microelectronic fabrication where particle contamination is not desired [3]. These low pressure systems are almost exclusively glow discharges having gradients in plasma densities that are small on the scale length of any given particle. In contrast, the diameter

3 Author to whom any correspondence should be addressed. of the streamer in atmospheric pressure discharges can be commensurate with the size of the particle. The dynamics of the propagation of streamers that intersect with particles can therefore be significantly perturbed.

The motivation for studying streamer interactions with particles comes from a variety of sources, including plasma aided combustion and functionalization of particles for biomedical applications. For example, high voltage initiated streamers in fuel-air mixtures are being investigated as a means of producing radicals to increase the flame speed $[4,5]$. These mixtures can contain both fuel vapour and fuel aerosols or droplets. The intersection of streamers with these aerosols can be expected to change the rate of radical generation by both changing the properties of the streamer and by transferring heat to the droplet, thereby increasing its rate of evaporation. (Note that gas heating in the streamer channel and enhanced droplet evaporation may occur during the latter stages of streamer evolution, for example, during the streamer-to-spark transition.) The purposeful functionalization of the surface of small particles for industrial and pharmaceutical purposes is also of interest 
where the ability to produce radicals inexpensively motivates the use of atmospheric pressure plasmas [6].

Although there has been significant progress in the development of 2-dimensional (2D) models for the propagation of streamers in uniform and non-uniform electric fields [7,8] there have been few investigations of the interactions of these streamers with particles, either experimentally or theoretically. One example is the investigation of the effects of dielectric liquid droplets on the electrodynamics of streamers in air at atmospheric pressure [9]. It was found that a jet of droplets can both guide the direction and alter the speed of the streamer.

Although the scale lengths are very different, the propagation of streamers along insulating surfaces has some relevance to the interaction of streamers with particles. In this case electron avalanche in front of the streamer head is enhanced by the presence of a dielectric due to electron emission from the surface produced by photons emitted by the discharge [10]. The higher rate of electron avalanche increases the speed of the streamer.

In this paper, we discuss results from a 2D computational investigation of the intersection of a positive corona streamer sustained in humid atmospheric pressure air with dielectric particles. We found that the intersection of a propagating streamer with a particle both polarizes and charges the particles to the local plasma potential. The polarization and charging produce local electric fields and sheaths around the particle that can significantly affect the local rate of ionization. If the capacitance of the particle is large enough, significant charge can be removed from the streamer. In such cases, the streamer can momentarily stall, only to be reinitiated on the opposite side of the particle. When this occurs in a series of aligned particles, the streamer may be handed off from one particle to another. For certain conditions these perturbations can result in streamer branching [11].

The model is described in section 2 and typical streamer characteristics in the absence of dust particles are discussed in section 3. The consequences of streamers interacting with isolated and multiple particles are discussed in sections 4 and 5 . Concluding remarks appear in section 6.

\section{Description of the model}

The physical processes and algorithms used in the model are discussed in detail in [12] and so will be briefly reviewed here. The model, nonPDPSIM, is a multi-fluid hydrodynamics simulation in which transport equations for all charged and neutral species and Poisson's equation are integrated as a function of time. The fundamental equations for charged species that are solved are the following:

$$
\begin{gathered}
-\nabla \cdot \varepsilon \nabla \Phi=\sum_{j} N_{j} q_{j}+\rho_{\mathrm{s}}, \\
\frac{\partial N_{j}}{\partial t}=-\nabla \cdot \vec{\phi}_{j}+S_{j}, \\
\frac{\partial \rho_{\mathrm{s}}}{\partial t}=\sum_{j} q_{j}\left(-\nabla \cdot \vec{\phi}_{j}+S_{j}\right)-\nabla \cdot(\sigma(-\nabla \Phi)),
\end{gathered}
$$

where $\varepsilon, \Phi, \rho_{\mathrm{s}}, N, \vec{\phi}, \sigma, S$ and $q$ are the permittivity, electric potential, surface charge density, species number density, species flux, conductivity of solid materials, source term and elementary charge respectively. Charged particle fluxes are given by the Scharfetter-Gummel formulation [13]. Poisson's equation (equation (1)), transport equations for conservation of the charged species $j$ (equation (2)) and the material and surface charge balance equation (equation (3)) are simultaneously integrated using a sparse-matrix, Newton iteration technique.

Updates of the charged particle densities and electric potential are followed by an implicit update of the electron temperature by solving the electron energy conservation equation,

$$
\frac{\partial\left(n_{\mathrm{e}} \varepsilon\right)}{\partial t}=\vec{j} \cdot \vec{E}-\nabla \cdot\left(\frac{5}{2} \varepsilon \vec{\phi}_{\mathrm{e}}-\kappa \nabla T_{\mathrm{e}}\right)-n_{\mathrm{e}} \sum_{i} \Delta \varepsilon_{i} k_{i} N_{i}
$$

where for electron density $n_{\mathrm{e}}$ the average electron energy $\varepsilon=$ $(3 / 2) k T_{\mathrm{e}}$ for electron temperature $T_{\mathrm{e}}, \phi_{\mathrm{e}}$ is the ScharfetterGummel form of the electron flux, $\vec{j}=q \vec{\phi}_{\mathrm{e}}$ is the total electron current and $\kappa$ is the electron thermal conductivity. The summation is over electron collisions with species having density $N_{i}$ and rate coefficient $k_{i}$ resulting in change in electron energy $\Delta \varepsilon_{i}$. The electron transport coefficients and rate coefficients for bulk electrons as a function of $T_{\mathrm{e}}$ are obtained by solving Boltzmann's equation for the electron energy distribution. As streamer propagation on time scales of only tens of nanoseconds are considered, we do not address the bulk hydrodynamic motion of the neutral gas though we do include diffusive transport of radicals.

The source term in equation (2) includes photoionization which accounts for the production of precursor electrons ahead of the streamer front. The photoionization is computed by producing Green's functions, which also account for view angles and obscurations produced by intervening electrodes or particles. The rate of photoionization is given by

$$
\begin{aligned}
\frac{\partial N_{i}^{+}(\vec{r})}{\partial t} & =\sum_{j} \int N_{j}\left(\vec{r}^{\prime}\right) A_{j} G\left(\vec{r}, \vec{r}^{\prime}\right) \sigma_{i j} \mathrm{~d}^{3} \vec{r}^{\prime} \\
G\left(\vec{r}, \vec{r}^{\prime}\right) & =\frac{\exp \left(-\sum_{k} \int_{\vec{r}^{\prime}} N_{k}\left(\vec{r}^{\prime \prime}\right) \sigma_{k j} \mathrm{~d} \vec{r}^{\prime \prime}\right)}{4 \pi\left|\vec{r}-\vec{r}^{\prime}\right|^{2}} .
\end{aligned}
$$

In these expressions, excited state $N_{j}$ emits a photon at location $\vec{r}^{\prime}$ with the rate given by Einstein coefficient $A_{j}$ and ionizes species $N_{i}$ with cross section $\sigma_{i j}$ at location $\vec{r}$. In traversing the plasma the photons are absorbed by species $N_{k}$ with cross section $\sigma_{k j}$. We assumed that photoionization occurs by absorption by oxygen molecules of radiation emitted by $\mathrm{N}_{2}\left(b^{1} \Pi\right)$ and $\mathrm{N}_{2}\left(b^{\prime 1} \Sigma\right)$ in the wavelength range 98-102 nm. The non-ionizing absorption cross section is approximated as $2 \times 10^{-17} \mathrm{~cm}^{-2}$ while that for the ionizing cross section is $1 \times 10^{-18} \mathrm{~cm}^{2}$. The precise value of these cross sections does not significantly affect the calculation other than in the speed of propagation of the streamer provided that the ionizing cross section is large enough to prevent the streamer from stalling. Photoemission of electrons by these photons incident on the surface of the dust particle is also included with a probability $\gamma=0.01$. Secondary electron emission by ion impact on all surfaces, including particles, was included with a probability of $\gamma=0.1$ 


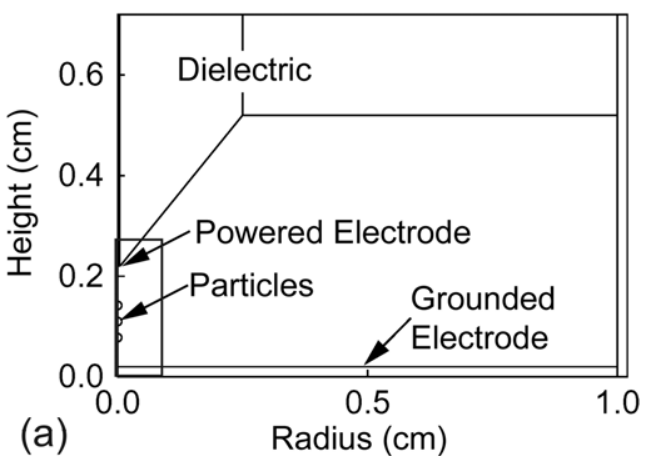

(a)

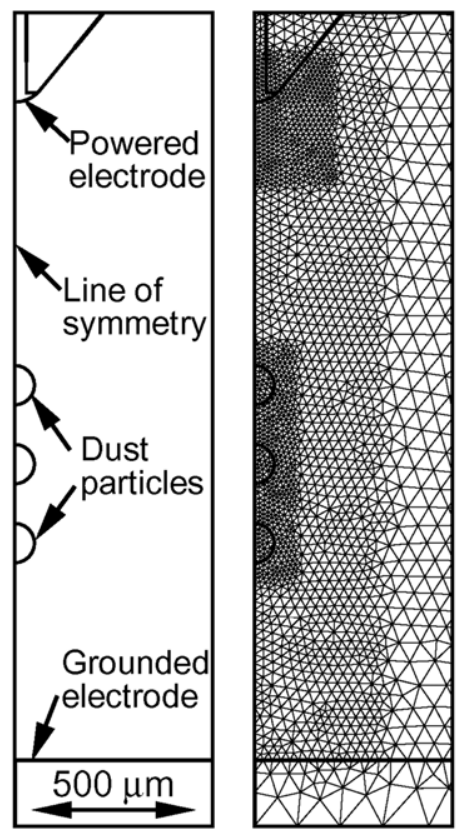

Figure 1. Schematic of the geometry. (a) Computational domain and $(b)$ close up of the anode-cathode gap. A typical numerical mesh is shown for three particles.

The gas mixture used for all cases is atmospheric pressure humid air $\mathrm{N}_{2} / \mathrm{O}_{2} / \mathrm{H}_{2} \mathrm{O}=79.5 / 19.5 / 1$ at $300 \mathrm{~K}$. The species included in the model are $\mathrm{N}_{2}, \mathrm{~N}_{2}(v), \mathrm{N}_{2}^{*}, \mathrm{~N}_{2}^{* *}, \mathrm{~N}_{2}^{+}, \mathrm{N}, \mathrm{N}^{*}$, $\mathrm{N}^{+}, \mathrm{N}_{4}^{+}, \mathrm{O}_{2}, \mathrm{O}_{2}^{*}, \mathrm{O}_{2}^{+}, \mathrm{O}_{2}^{-}, \mathrm{O}^{-}, \mathrm{O}, \mathrm{O}^{*}, \mathrm{O}^{+}, \mathrm{O}_{3}, \mathrm{H}_{2} \mathrm{O}, \mathrm{H}_{2} \mathrm{O}^{+}, \mathrm{H}_{2}$, $\mathrm{H}, \mathrm{OH}$ and electrons. The states $\mathrm{N}^{*}$ and $\mathrm{N}^{* *}$ are nominally $\mathrm{N}_{2}\left(\mathrm{~A}^{3} \Sigma\right)$ and $\mathrm{N}_{2}\left(b^{1} \Pi, b^{\prime 1} \Sigma\right)$ though the latter is treated as a lumped state including transitions higher than $\mathrm{N}_{2}\left(\mathrm{~A}^{3} \Sigma\right)$. $\mathrm{N}^{*}$ is nominally $\mathrm{N}\left({ }^{2} \mathrm{D}\right)$ but is also intended to be a lumped state for higher excitation as well. The states $\mathrm{O}^{*}$ and $\mathrm{O}_{2}^{*}$ are $\mathrm{O}\left({ }^{1} \mathrm{D}\right)$ and $\mathrm{O}_{2}\left({ }^{1} \Delta\right)$ and are also intended to be lumped states. The reaction mechanism used is identical to that discussed in [14]. To initiate the streamer, a small spot of a seed-charges (electrons and $\mathrm{N}_{2}^{+}$) was placed at $10 \mu \mathrm{m}$ from the tip of the anode. The seed plasma had a diameter of $250 \mu \mathrm{m}$ and a peak density of $1 \times 10^{8} \mathrm{~cm}^{-3}$. There is otherwise no initial plasma density elsewhere in the computational domain.

The model geometry is shown in figure $1(a)$. The positive corona discharge is sustained between a rod encased in a dielectric $\left(\varepsilon / \varepsilon_{0}=5\right)$ having an exposed edge with a radius of curvature of $0.07 \mathrm{~cm}$ as shown in figure $1(b)$. The electrode is charged to $15 \mathrm{kV}$ and is separated from a flat grounded metal surface by $0.2 \mathrm{~cm}$. The numerical grid uses an unstructured mesh with triangular elements with different refinement regions in order to resolve the detail of the electrode tip and the fine structure of dust particles as well as larger surrounding features. The schematic shown in figure $1(b)$ is the meshing used for a case with three dust particles. The dust particle radii we investigated are in the range of $25-80 \mu \mathrm{m}$.

The dielectric properties of the particles were specified and the interiors of the particles (as well as all solid materials) were included in the computational domain. Poisson's equation (equation (1)) was solved throughout the entire computational domain (except in metals where the potential is specified as a boundary condition). Continuity equations for gas phase charged and neutral particles are only solved for in the plasma region. Equation (3) for surface and volume charges is solved on and inside all non-metallic materials. The collection of charges on plasma-dielectric interfaces and current continuity is a natural outcome of the method. As such, no unique boundary conditions are required for the interfaces of, for example, plasma and dielectrics. Although not used in this particular study, using this methodology, electrically floating materials can be treated as dielectrics having a sufficiently high conductivity and dielectric constant to have a negligible voltage drop across the material.

For the cases discussed here, the mesh consisted of approximately 5000-7000 nodes, of which about 4000-6000 are in the plasma region. The mesh resolution is $1-4 \mu \mathrm{m}$ near the anode and dust particles. The resolution of the mesh was chosen as a tradeoff between computer time required to run the case and resolving the physical phenomena. The resolution of the mesh was varied to confirm that predicted streamer properties, such as the diameter of the streamer, do not significantly change. The geometry is symmetric across the centre line.

Parametrizations were conducted to verify that the overall resolution of the mesh and the change in resolution between refinement zones of the mesh did not significantly affect the outcome of the calculation. For example, the entire mesh through which the streamer might propagate was replaced with a finer mesh having the minimum resolution shown in figure $1(b)$. The resulting propagation speed changed by only a few per cent, a change that was deemed acceptable.

\section{Typical streamer characteristics without particles}

As a point of departure, the properties of positive corona streamers in the absence of particles will be reviewed. Typical electrical and plasma characteristics at three successive times are shown in figures 2 and 3. A streamer is initiated in the high electric field near the anode where the small radius of curvature produces geometric electric field enhancement. The streamer then moves towards the cathode, against the direction of electron drift, by seeding electrons ahead of the streamer by photoionization. The streamer is a narrow conducting plasma channel which can be divided into the head where the electric field is large due to charge separation and where ionization occurs; and the low electric field channel connecting the head with the anode. Electrons and negative ions in the channel flow towards the anode (top) while positive ions flow slowly towards the cathode (bottom). The plasma in 


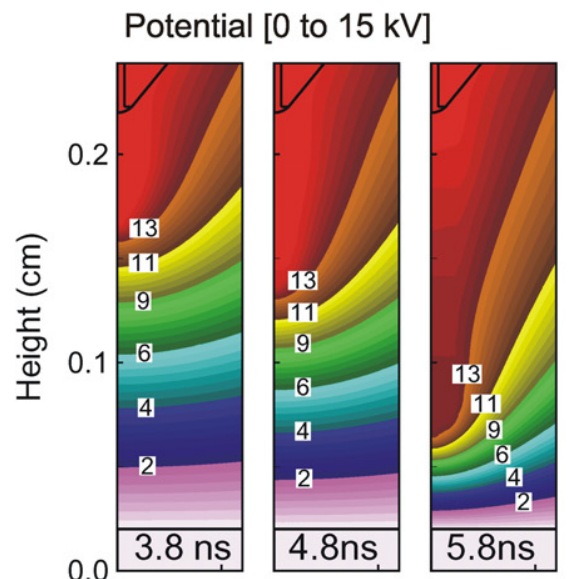

(a)

E/N [100 to $1000 \mathrm{Td}$, log scale]
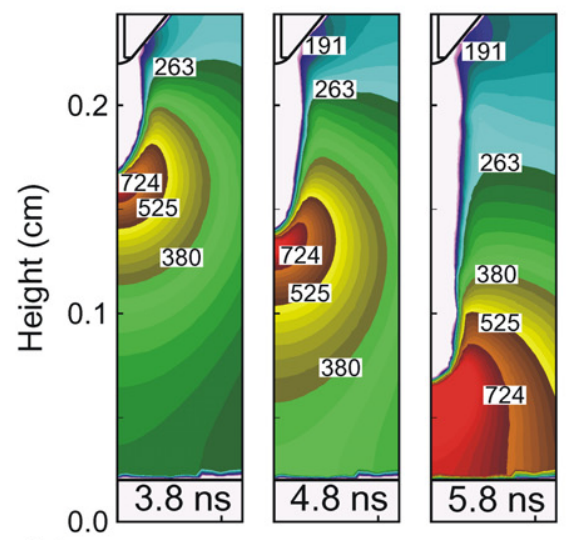

(b) $[\mathrm{e}] \times 10^{13} \mathrm{~cm}^{-3}$

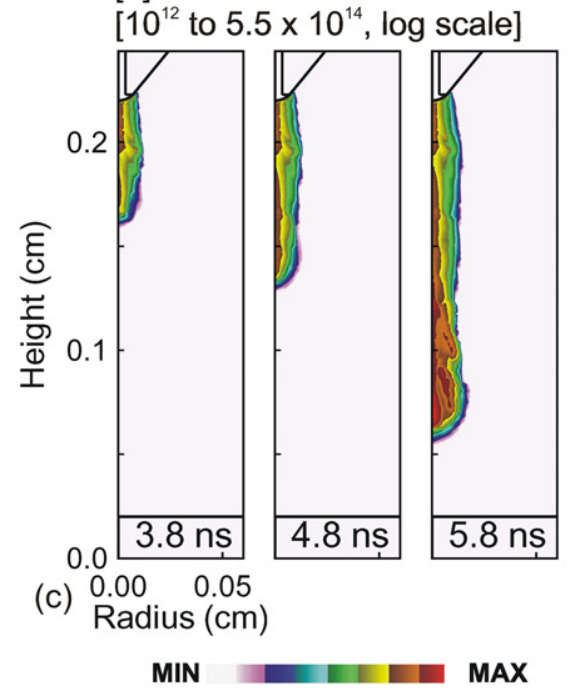

Figure 2. Typical streamer characteristics in atmospheric pressure humid air at 3.8, 4.8 and $5.8 \mathrm{~ns}$ after the start of the $15 \mathrm{kV}$ pulse. (a) Potential, $(b)$ reduced electric field and $(c)$ electron density. The ranges of plotted values are noted above each frame. The plots are symmetric across the left axis. The avalanche begins at the anode and propagates with a speed of $\approx 2 \times 10^{7} \mathrm{~cm} \mathrm{~s}^{-1}$.

the inner part of the channel is quasi-neutral with densities exceeding $5 \times 10^{14} \mathrm{~cm}^{-3}$. Positive space charge is concentrated at the axial tip (or head) of the streamer and at the radial boundaries, as shown in figure $3(a)$. The space charge at the radial boundaries produces a radially directed ambipolar
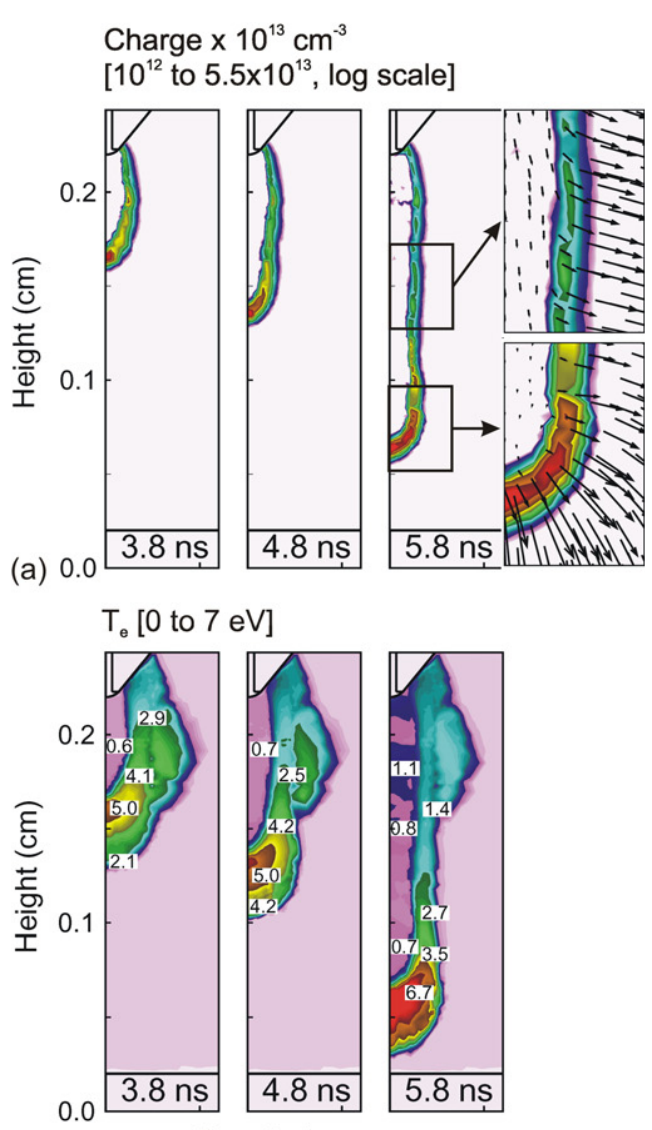

(b) $\mathrm{S}_{\mathrm{e}} \times 10^{22} \mathrm{~cm}^{-3} \mathrm{~s}^{-1}$

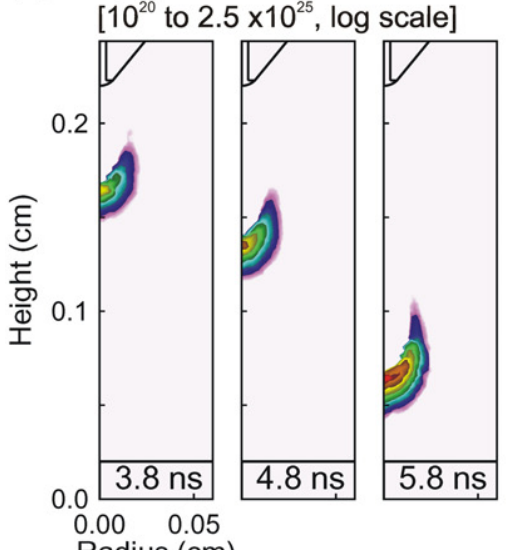

(c)

MIN

MAX

Figure 3. Typical streamer characteristics for the conditions of figure 2. (a) Positive space charge, $(b)$ electron temperature and (c) electron impact ionization source $S_{\mathrm{e}}$. Positive space charge is concentrated at the streamer boundaries. The electron temperature is high at the streamer tip, falling below $1 \mathrm{eV}$ in the streamer channel. The peaks in $S_{\mathrm{e}}$ indicate the location of the ionization front that propagates from the anode to the cathode. The magnified insets in figure 3(a) show the space charge with superimposed electric field vectors. These radially directed electric field vectors produce ambipolar forces which confine the plasma.

electric field that confines the plasma. These electric field vectors are shown by the insets in figure $3(a)$. The electric field vectors point downwards in the plasma channel and at the head of the streamer in the direction of net current flow. The 
electric field vectors point outwards on the channel boundaries and so produce the confining ambipolar forces.

The space charge at the head of the streamer produces electric fields exceeding $250 \mathrm{kV} \mathrm{cm}^{-1}$, or a normalized electric field of $E / N$ (electric field/gas number density) exceeding $1000 \mathrm{Td}\left(1 \mathrm{Td}=10^{-17} \mathrm{~V} \mathrm{~cm}^{2}\right)$. These large electric fields heat the electrons to temperatures as high as $7 \mathrm{eV}$, which in turn produce ionization at rates exceeding $10^{25} \mathrm{~cm}^{-3} \mathrm{~s}^{-1}$ thereby propagating the streamer. Ionization by electron impact occurs almost exclusively at the head of the streamer as shown in figure $3(c)$ as the low electric fields in the channel of the streamer $(E / N<20-30 \mathrm{Td})$ are insufficient to produce ionization significantly greater than attachment. Photon generation occurs both in the streamer head and in the conducting channel due to the finite lifetime of the excited states. Photoionization occurs in the plasma channel, in the streamer head and ahead of the streamer. It is only a significant and dominant source of ionization ahead of the streamer where it provides seed electrons to enable propagation of the streamer towards the cathode while the direction of net electron drift is towards the anode. Computationally, if photoionization is eliminated and in the absence of an initial distribution of preionization, the streamer will stall and fail to propagate.

The diameter of the streamer is an important consideration in dust particle-streamer interactions. Experimental observations [15] and numerical modelling have shown that the streamer radius is $100-200 \mu \mathrm{m}$, as we also obtain here. The propagation time of the streamer across the $2 \mathrm{~mm}$ gap is $3-4 \mathrm{~ns}$, resulting in speeds of $5 \times 10^{7} \mathrm{~cm} \mathrm{~s}^{-1}$.

Streamer characteristics along the axis are shown in figure 4. The streamer propagates from right to left in the figure. The head of the streamer is denoted by the peak in the electric field and in the space charge. The potential drop across the channel behind the streamer head is small, which results in the majority of the applied voltage being dropped between the head of the streamer and the ground plane. As the streamer propagates and extends the high conductivity channel, the applied voltage is dropped across a successively shorter distance, thereby increasing the electric field ahead of the streamer. The positive space charge that is concentrated in the narrow layer on the streamer boundary produces a high electric field in front of the streamer and, at the same time, shields the electric field from penetration into the streamer body. The peak $E / N$ in streamer head increases from 750 to $1300 \mathrm{Td}$ as the streamer propagates. The electron density behind the streamer front is $10^{13}-10^{14} \mathrm{~cm}^{-3}$.

\section{Streamer dynamics intersecting with a single particle}

One of the most important phenomena governing the dynamics of streamers interacting with particles is the polarization of the dielectric particle in an external electric field. Although the electric field in a point-to-plane corona and in the vicinity of the streamer head can be highly nonuniform, it is instructive to first review the polarization of a dielectric particle in a uniform external electric field. For a dielectric sphere of constant permittivity $\varepsilon$ with radius $r_{0}$ placed in a uniform external electric field $\vec{E}_{0}$ that is aligned with the polar axis,
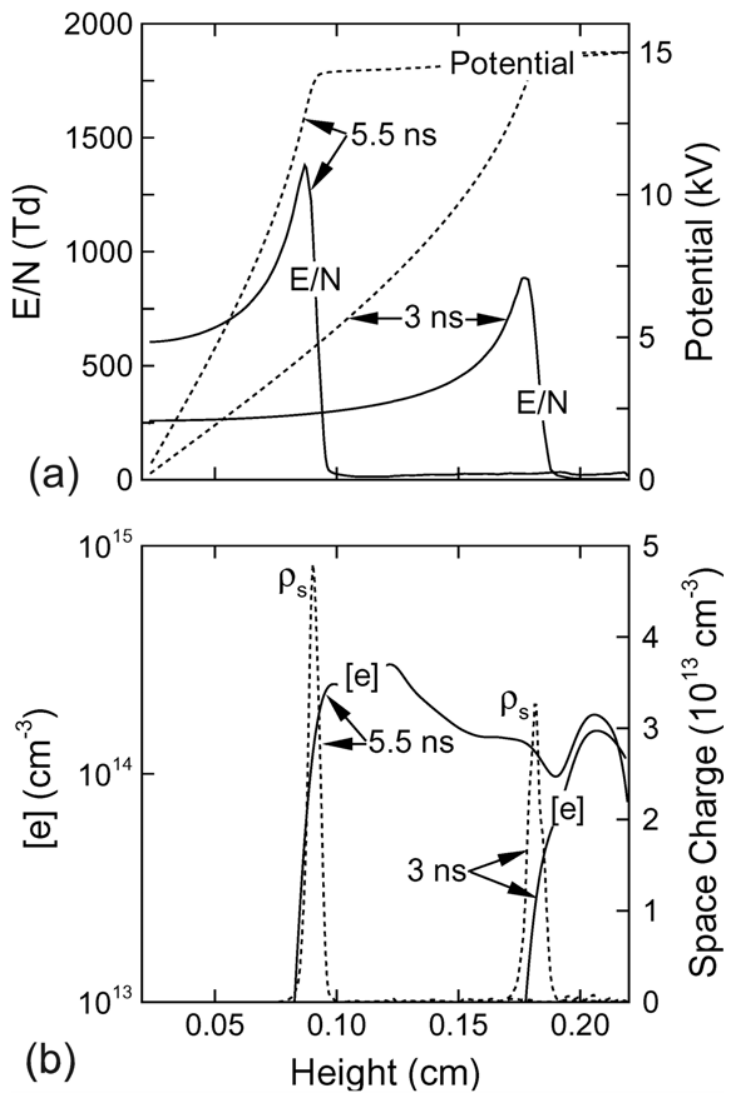

Figure 4. Streamer characteristics along the axis at $t=3$ and $5.5 \mathrm{~ns}$. (a) Reduced electric field (- ${ }^{-}$and potential (- - - ) and (b) electron density (-) and positive space charge (- - -). The $E / N$ in the channel behind the streamer is low enough so that net ionization is not significant.

the polarization field is [16]

$$
\begin{aligned}
\vec{E}= & E_{0} \cos \theta\left[1+2\left(\frac{r_{0}}{r}\right)^{3} \frac{\varepsilon-1}{\varepsilon+2}\right] \hat{a}_{r} \\
& -E_{0} \sin \theta\left[1-\left(\frac{r_{0}}{r}\right)^{3} \frac{\varepsilon-1}{\varepsilon+2}\right] \hat{a}_{\theta} .
\end{aligned}
$$

The electric field at the poles $(\theta=0, \pi)$ of the particle is enhanced by a factor of $1+2(\varepsilon-1) /(\varepsilon+2)$ compared with the unperturbed external electric field. At the equator $(\theta=\pi / 2)$ the electric field at the surface of the particle is decreased by a factor of $1-(\varepsilon-1) /(\varepsilon+2)$ compared with the applied field. The corresponding values for a conducting sphere can be obtained by allowing $\varepsilon \rightarrow \infty$. In this case the electric field is enhanced by a factor of 3 near the poles of the sphere and is zero near the equator. These results indicate that even in a uniform electric field, the polarization of the particle may produce non-uniform rates of ionization as a function of azimuth. Note that an expression analogous to equation (6) cannot in general be obtained analytically if the electric field is non-uniform and transient. As such, equation (6) is not directly used in the model. In the model, the polarization electric fields are a natural outcome of the solution of Poisson's equation (equation (1)) in both the plasma and the particles while including all of the appropriate material properties and charges.

The reduced electric field around a particle $\left(\varepsilon / \varepsilon_{0}=5\right.$, $60 \mu \mathrm{m}$ radius) in a uniform electric field of $100 \mathrm{Td}$, as obtained 


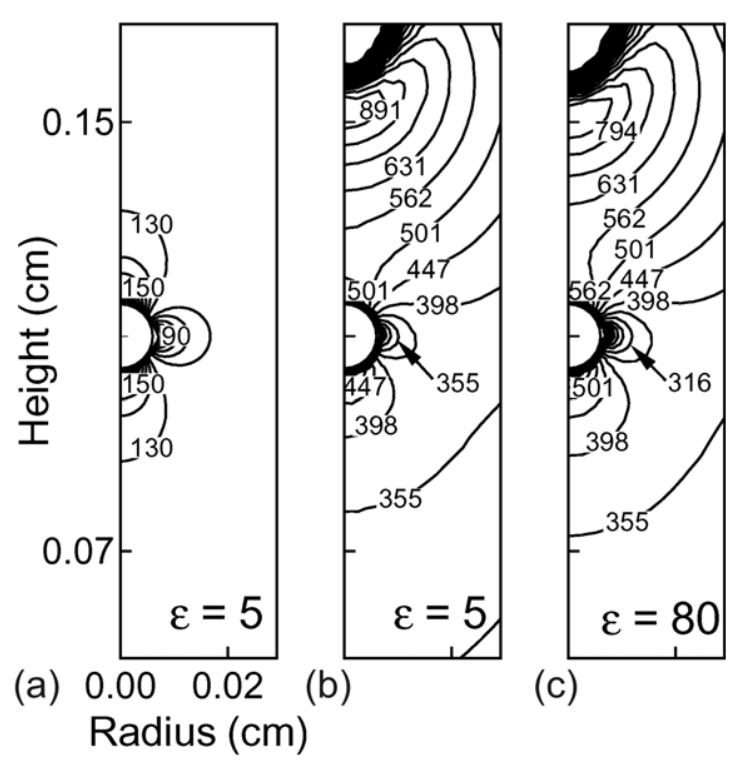

Figure 5. Electric field (Td) around dielectric particles $60 \mu \mathrm{m}$ radius. (a) A particle with $\varepsilon / \varepsilon_{0}=5$ in a uniform electric field of $100 \mathrm{Td}$ between parallel plate electrodes. $(b)$ Particle with $\varepsilon / \varepsilon_{0}=5$ in a non-uniform electric field resulting from the approach of a streamer tip (at the top of the frame). (c) Particle with $\varepsilon / \varepsilon_{0}=80$ as a streamer tip approaches. The field at the equator is substantially lower than at the poles of particles. The large electric field in the tip of the approaching streamer intensifies the polarization of the particle.

with the model, is shown in figure $5(a) . E / N$ near the particle in the non-uniform field of an approaching streamer tip is shown in figure $5(b)$. In both the cases the electric field at the equator of the particles is substantially lower than at the poles. In the non-uniform field produced by an approaching streamer tip, the field enhancement at the pole which faces the streamer is larger than at the opposite side, an effect that is more pronounced for particles having a larger permittivity, as shown in figure 5(c). In the absence of other effects, and assuming the spatial extent of the electric field enhancement is larger than the mean free path of electrons, this electric field enhancement can result in significant heating of electrons at the poles of the particles and so enhance ionization processes there.

Particles having large permittivities, as shown in figure $5(c)$, are of interest to, for example, streamers propagating through an aerosol of a polar liquid. Water is one such highly polar liquid having $\varepsilon / \varepsilon_{0}=80$ and with a nonzero electrical conductivity $\sigma$. Water exposed to an electric field $E$ for a time $t$ behaves as a dielectric if $t \ll \varepsilon / \sigma$ while for $t \gg \varepsilon / \sigma$ it behaves as an ion semiconductor. For typical conditions $\left(\sigma=100 \mu \mathrm{S} \mathrm{cm}^{-1}\right) \varepsilon / \sigma=72 \mathrm{~ns}$ which is large compared with the time for streamer interactions with particles of tens of micrometre in size. As a result, in the vast majority of applications of streamers propagating through water aerosols, water droplets behave as dielectrics.

Plasma properties (electron temperature, electron ionization source and electron density) in the vicinity of a particle $60 \mu \mathrm{m}$ in radius and with $\varepsilon / \varepsilon_{0}=80$ are shown in figure 6 . The streamer-particle interaction proceeds in two stages. During the first stage, the streamer front approaches the top of the particle. A strong interaction occurs between the already large electric field at the streamer tip and the enhanced local field near the poles of the particle due to polarization. This results in an elevated electron temperature $(7.5 \mathrm{eV})$ and rapid ionization. In the second stage, the plasma intersects with the particle. The particle is charged positively on the top surface and eventually negatively on the bottom surface. As the plasma envelopes the particle, first from the top, the region of electric field enhancement rotates around the particle producing a high $T_{\mathrm{e}}$ and ionization that also rotates around the particle. Electrons flowing upwards towards the anode produce an electron deficient wake above the particle.

As discussed below, there is a deficiency of electrons near the equator of the particle for at least two reasons. The first is that on average there is a smaller electric field near the equator due to polarization of the particle. The second is that as the plasma begins to propagate around and envelope the particle, photoionization seeds electrons in the higher electric field near the bottom of the particle. This preionization, in a sense, launches a new streamer from the bottom of the particle. The new streamer is launched without having to fully develop the plasma at the equator to electrically connect with the streamer intersecting at the top of the particle.

The net negative and positive charge densities, both in the plasma and on the particle, are shown in figure 7 as a streamer approaches and passes a particle. The directions of the electric field vectors are also shown. The tip and the radial boundaries of the streamer are regions of net positive charge. The channel of the streamer is essential quasi-neutral. When the streamer arrives at the uncharged particle the positive space charge of the streamer tip is deposited on the upper surface of the particle surface. In this regard, the particle acts as a solid capacitor, an electrical obstacle, in the path of the streamer. The particle removes charge from the streamer, slowing it and in some cases preventing the streamer from further propagation. Due to the net electron flow being upwards towards the anode, the upper surface of the particle retains a net positive charge. As the plasma propagates around and develops beneath the particle due to seeding by photoionization, electron flow upwards towards the anode negatively charges the bottom of the particle. The adjacent plasma retains a net positive charge, thereby making the bottom of the particle a cathode-like surface.

The net direction of the electric field is downwards. As the streamer tip arrives, the particle is highly polarized (frames for $3.8 \mathrm{~ns}$ in figure 7) resulting in the electric field vectors focusing at the poles and pointing outwards at the equator. As the streamer intersects with (frames for $4.2 \mathrm{~ns}$ ) and passes (frames for $4.6 \mathrm{~ns}$ ) the particle, there is net current flow around the particle, as indicated by the direction of the electric field vectors near the equator that sweeps around the particle. The net positive charge remaining in the plasma at the equator reverses the direction of the electric field to point towards the particle. The net positive charge at the radial boundaries of the streamer produce ambipolar fields indicated by the radially pointing vectors. During positive charging of the top of the particle (frame for $3.8 \mathrm{~ns}$ in figure $7(a)$ ), the electric field vectors point from the streamer head towards the particle. After the streamer passes, leaving a quasi neutral plasma behind, the electric field is weakened on the top of the particle due to the accumulated positive charge but still points towards the particle in the direction of the net electric field. Due to the negative 


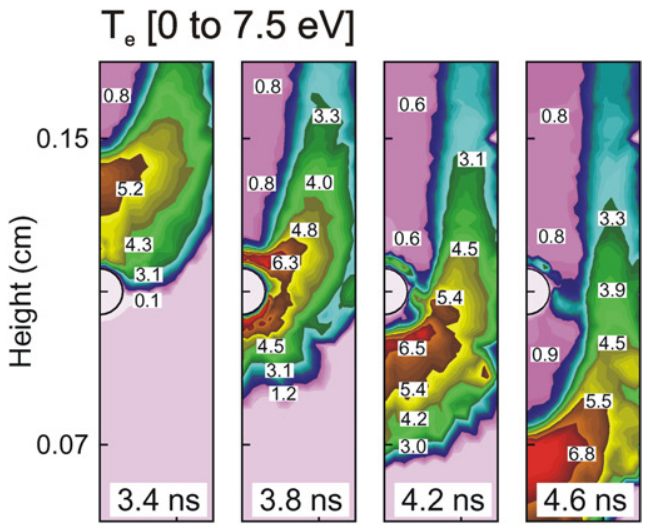

(a) $\mathrm{S}_{\mathrm{e}} \times 10^{22} \mathrm{~cm}^{-3} \mathrm{~s}^{-1}$

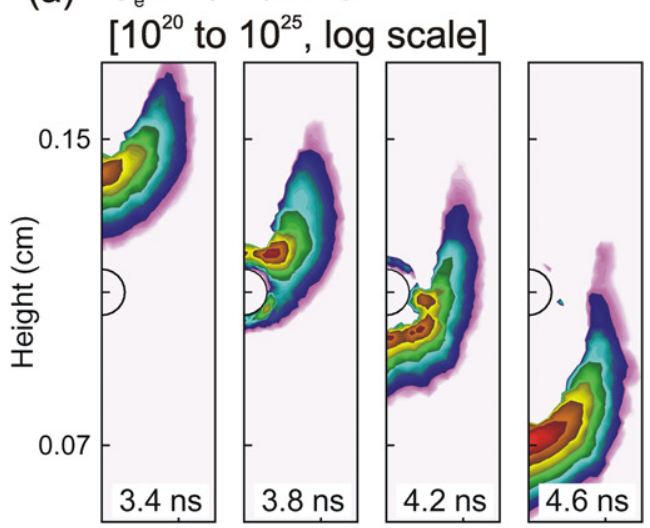

(b) $[\mathrm{e}] \times 10^{13} \mathrm{~cm}^{-3}$



Figure 6. Plasma characteristics around a $60 \mu \mathrm{m}$ dust particle $\left(\varepsilon / \varepsilon_{0}=80\right)$ as a function of time after initiation. (a) Electron temperature, $(b)$ electron impact ionization source and $(c)$ electron density. The streamer is stalled for about $0.4 \mathrm{~ns}$ (two middle frames) as it charges the particle. The plasma propagates around and envelopes the particle and is then rapidly reinitiated as a new streamer from the bottom of the particle.

charging on the bottom of the particle, the electric field vectors point upwards towards the particle (frames for $4.6 \mathrm{~ns}$ ).

Note that the particle charging occurs within $0.8 \mathrm{~ns}$ due to both the large current density available from the streamer and the short transit time of the streamer across the particle. The process is more rapid than the charging of a dust grain, tens to
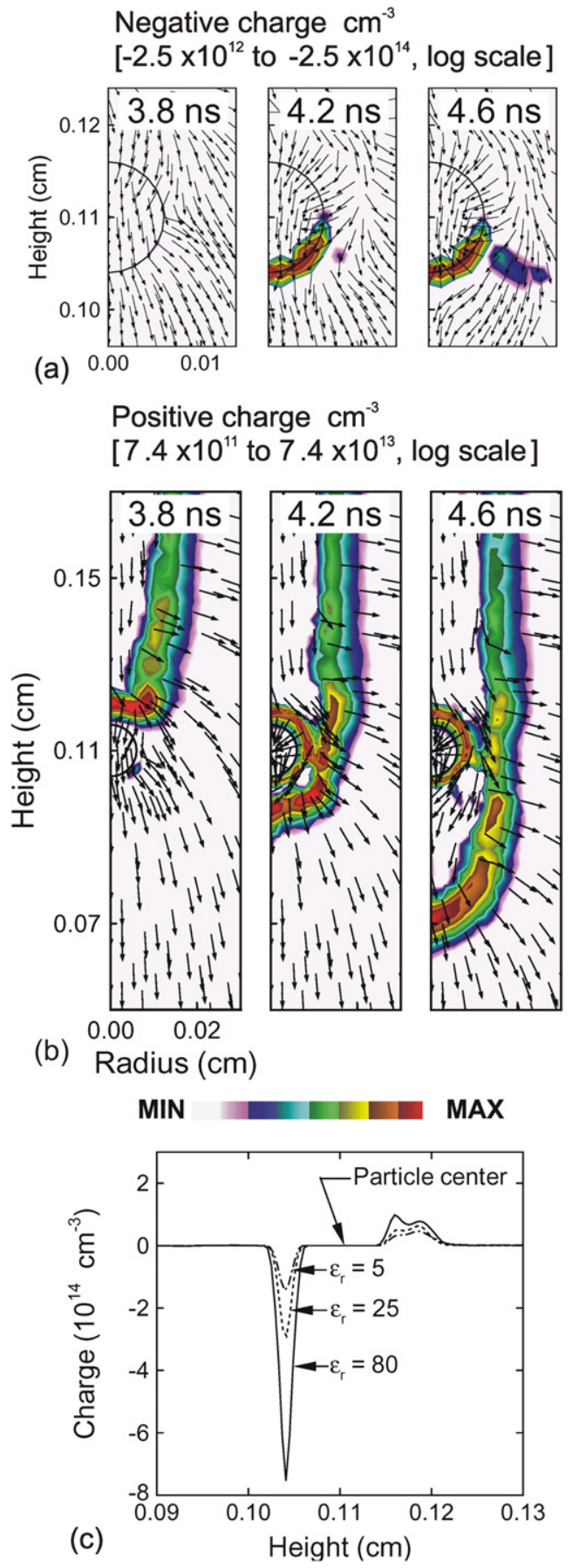

Figure 7. The process of particle charging for a $60 \mu \mathrm{m}$ dust particle $\left(\varepsilon / \varepsilon_{0}=80\right)$. (a) Net negative and $(b)$ net positive space charge with superimposed electric field vectors. The arrows show only the direction of the electric field. (c) Charge density along the axis in the gas phase and accumulated on the particle body as a function of particle dielectric constant. The charge increases with increase in the dielectric constant. Streamer propagation is from top to bottom for figures $7(a)$ and $(b)$ and right to left for figure $7(c)$.

hundreds of nanoseconds, in an initially quasi-neutral plasma where particle fluxes are ambipolar limited [17].

To the degree that the particle acts as a capacitor, it will charge until its potential equilibrates with the local plasma potential, offset by the floating potential required to balance charge particle fluxes. The charge on the particle required to 
$\mathrm{E} / \mathrm{N}[100$ to $1000 \mathrm{Td}$, log scale]
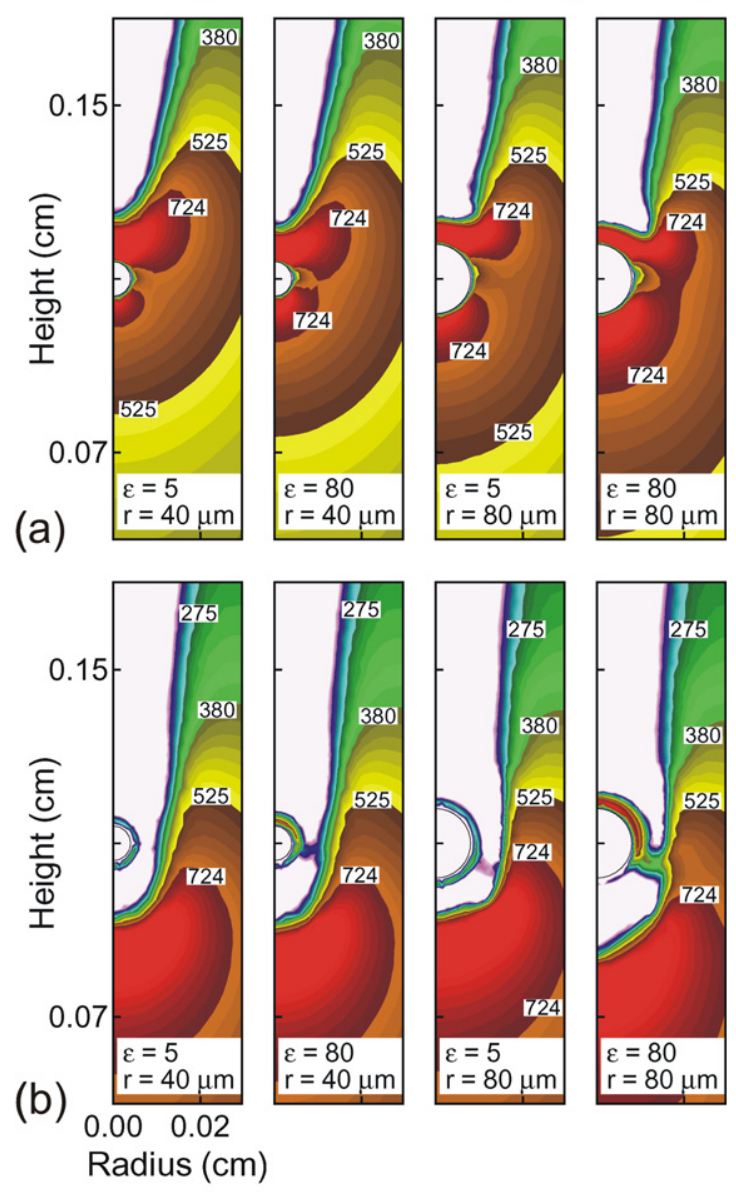

MIN

Figure 8. Streamer-particle interaction as a function of particle size and dielectric constant. (a) Reduced electric field at $t=3.8 \mathrm{~ns}$ before the streamer arrives at the particle and $(b)$ reduced electric field at $t=4.4 \mathrm{~ns}$ after the streamer arrives at the particle. Contour labels are in Td. Small particles or particles with low dielectric constant are enveloped by the streamer plasma. Particles with high dielectric constant stall and reinitiate the streamer.

achieve this balance increases with increasing permittivity as the capacitance of the particle increases. For example, the charge density on the axis for particles having $\varepsilon / \varepsilon_{0}=5,25$ and 80 are shown in figure $7(c)$. More charge resides on the particle with increasing $\varepsilon / \varepsilon_{0}$ in order to raise the potential of the particle to the plasma potential.

Electric field magnitudes as a function of particle size and dielectric constant are shown in figure 8 as the streamer approaches the particle (3.8 ns) and after passing the particle $(4.4 \mathrm{~ns})$. Although the basic features of the streamer-particle interactions are the same, there is a dependence on the size, conductivity and permittivity of the particle. Particles with small capacitances (small particles and/or low permittivity) are enveloped by the streamer. Particles with large capacitance (large particle and/or high dielectric permittivity) can stall and relaunch the streamer.

When the streamer approaches the particle, polarization creates a large electric field on the top side. The streamer head maintains a convex shape. Ionization in this large electric field
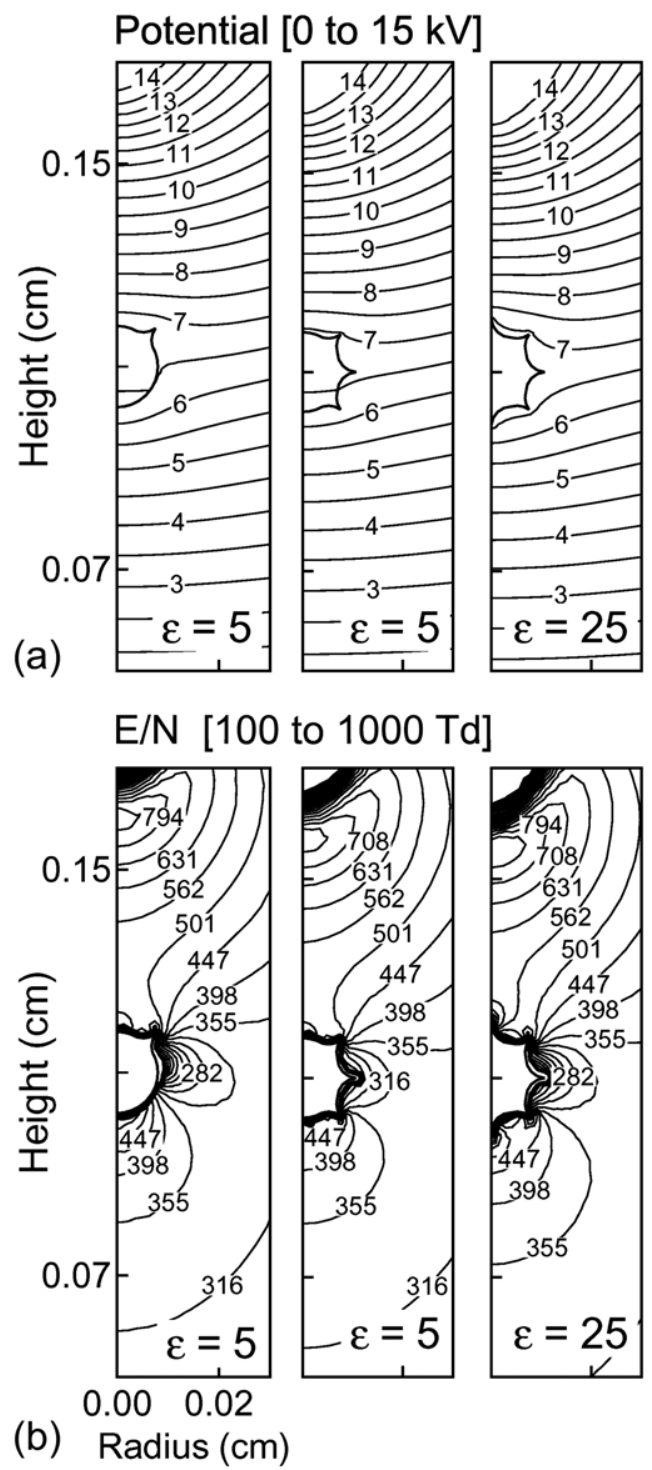

Figure 9. Electrical properties for a $80 \mu \mathrm{m}$ dielectric particle with sharp features at $t=3.05 \mathrm{~ns}$ as the streamer tip approaches the particle. (a) Potential and $(b)$ reduced electric field. Sharp edges enhance the electric field in the direction of streamer propagation.

then provides a ready source of plasma to begin charging the particle. If the capacitance of the particle is small enough, the particle rapidly charges and the plasma rotates around the particle, completely enveloping it. This is the case for the $40 \mu \mathrm{m}$ particle with $\varepsilon / \varepsilon_{0}=5$ in figure 8 .

For a large particle $\left(80 \mu \mathrm{m}, \varepsilon / \varepsilon_{0}=80\right)$ the streamer stalls above the particle as charge is removed from the streamer head to charge the particle. The streamer slowly propagates around the particle, weakening the electric field at its boundary, producing a concave shape to the streamer head. The bottom of the particle is initially geometrically shadowed from the plasma. As the plasma rotates around the particle, photons produced in the plasma begin to illuminate the gas directly below the particle. If the polarization of the particle and electric fields at the bottom of the particle are sufficiently large, the photoionization resulting from the illumination seeds an avalanche. A streamer is then launched from the bottom of the particle. In particularly severe cases, the streamers 


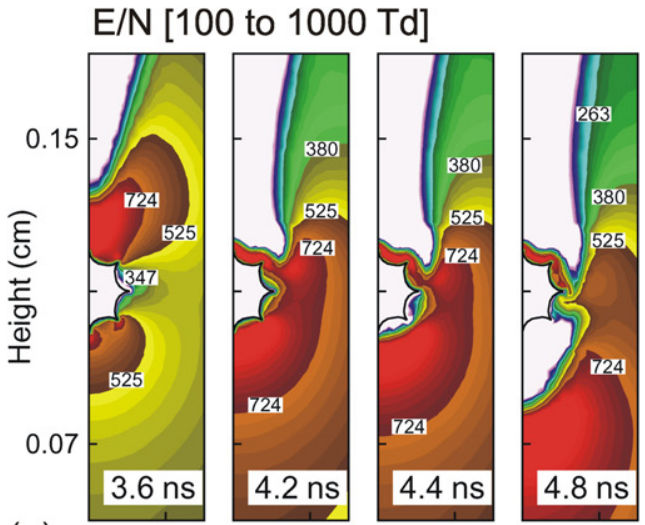

(a)

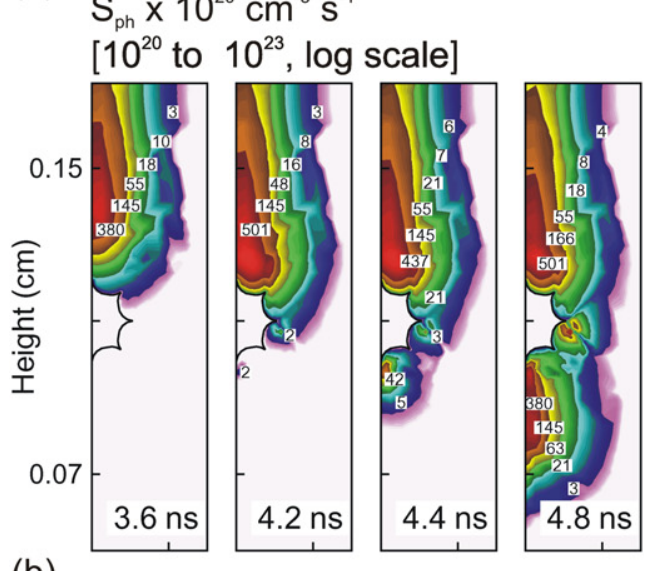

(b)

$$
\begin{aligned}
& {[\mathrm{e}] \times 10^{13} \mathrm{~cm}^{-3}} \\
& {\left[10^{12} \text { to } 4 \times 10^{14} \mathrm{~cm}^{-3}\right]}
\end{aligned}
$$
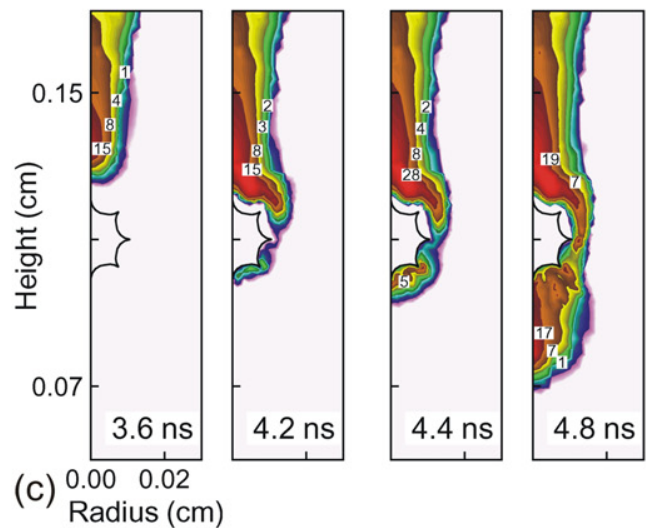

MIN

MAX

Figure 10. Plasma characteristics around a $80 \mu \mathrm{m}$ dielectric particle $\left(\varepsilon / \varepsilon_{0}=5\right)$ with sharp features as a function of time. ( $\left.a\right)$ Reduced electric field, $(b)$ photoionization source and $(c)$ electron density. The streamer is stalled for $0.6 \mathrm{~ns}$ (two middle frames) waiting for precursor photoelectrons to be produced at the bottom part of the particle.

above and below the particle may not be physically connected by a conductive plasma channel. This is demonstrated by the large particle $\left(80 \mu \mathrm{m}, \varepsilon / \varepsilon_{0}=80\right)$ in figure $8(b)$. The conductive plasma channel is denoted by the region where $E / N<100 \mathrm{Td}$. For this particle, $E / N$ greatly exceeds $100 \mathrm{Td}$ at the equator. A second streamer is launched from the bottom of the particle without there being a conductive channel completely surrounding the particle.
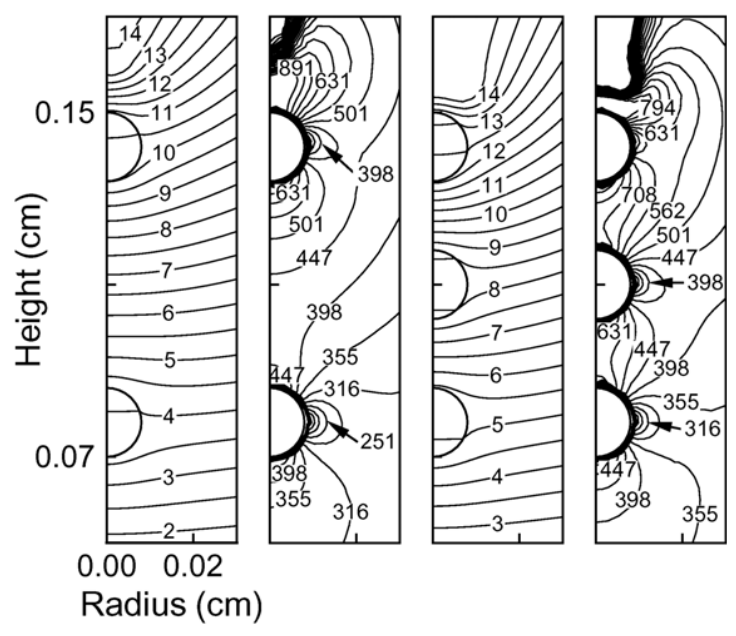

Figure 11. Contours of potential and reduced electric field for two and three dielectric particles of $80 \mu \mathrm{m}$ radius $\left(\varepsilon / \varepsilon_{0}=5\right)$ as the streamer tip approaches the particles. The particles act as a bridge that extends the region of the enhanced electric field due to polarization towards the cathode.

Dust particles are often not spherical and so, depending on conductivity and permittivity, electric field enhancement may significantly affect the particle-streamer interaction. For example, the electric potential and electric field near an $80 \mu \mathrm{m}$ particle with sharp features are shown in figure 9 as the streamer tip approaches the particle. The polarization and subsequent electric field enhancement produced by the approaching streamer is magnified by the geometric enhancement of the sharp points of the particles. These enhancements increase the likelihood that at these points new streamers are formed and launched.

The electric field, source of photoionization and electron density are shown in figure 10 as the streamer approaches and passes a particle having sharp tips. The streamer slows as it approaches the particle $(t=3.6 \mathrm{~ns})$ and is nearly stalled for $0.6 \mathrm{~ns}$ (two middle frames). The increased capacitance of the small structures of the particle requires so much current from the streamer to charge that the streamer is unable to propagate. As the particle locally charges, the streamer propagates and rotates around the particle, with its head replicating the shape of the particle. The sharp features of the particle obscure and shadow photoionizing radiation from reaching the bottom of the particle where the electric field is large by both polarization and geometric enhancement. As the plasma rotates beyond the equator and the photon source is no longer shadowed, photoionization occurs in the high electric field region at the downward pointing tip on the bottom of the particle. This seeds the launching of a new streamer from the bottom of the particle. The new streamer has a time delay of $0.6 \mathrm{~ns}$ from when the primary streamer intersected the top of the particle while the streamer waits for photoelectrons to appear at the bottom of the particle.

When the lower streamer is launched, there is at best a tenuous plasma channel between the upper and lower streamers. This is perhaps best illustrated by the $E / N$ values at $4.8 \mathrm{~ns}$. Fully developed plasma channels have small values of $E / N$ due to their high conductivity. The lack of bridging by dense plasma between the first (top) and second (bottom) 




(a)

\section{$\mathrm{T}_{\mathrm{e}}[1$ to $7.5 \mathrm{eV}]$}
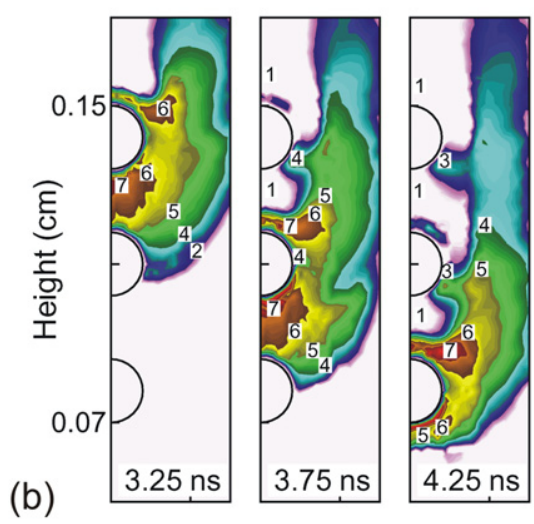

MIN

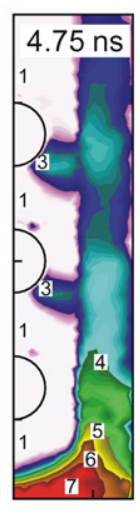

MAX (c)

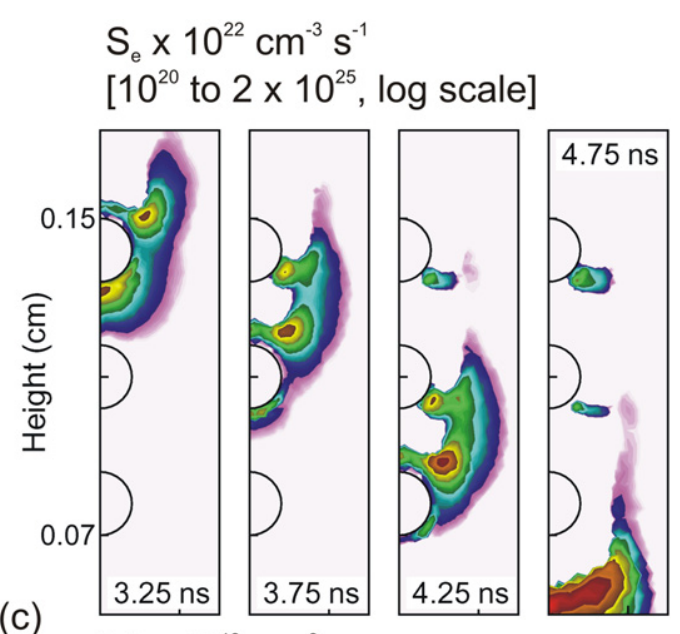

[e] $\times 10^{13} \mathrm{~cm}^{-3}$

$\left[10^{10}\right.$ to $6 \times 10^{14}$, log scale]

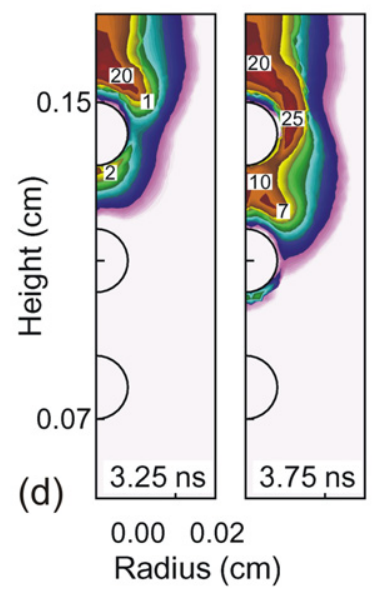

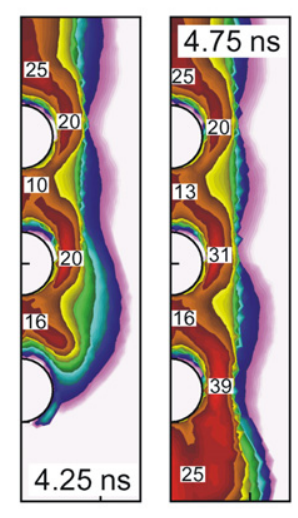

Figure 12. Plasma characteristics around three $80 \mu \mathrm{m}$ dust particles $\left(\varepsilon / \varepsilon_{0}=5\right)$ as a function of time. (a) Reduced electric field, $(b)$ electron temperature, $(c)$ electron impact ionization source and $(d)$ electron density. The upper particles reinitiate the streamer while the bottom particle is enveloped by the streamer plasma.

streamers that would produce a low $E / N$ indicates the lack of continuity. In this case, the delay between intersection of the upper streamer and launching of the lower streamer results in a substantial decrease in the average streamer velocity compared with a streamer not intersecting a particle.

\section{Streamer interactions with multiple particles}

Streamers propagating through dense smoke or fog may, statistically, encounter particles that are close enough together so that their interactions with the particles are coupled. To investigate the consequences of streamers interacting with multiple particles, we simulated streamers incident on two and three aligned particles while varying their size and dielectric constant. We found that particles in close proximity can act as a bridge that elongates the region of the enhanced electric field resulting from polarization towards the cathode. For example, the electric potential and electric field are shown in figure 11 for assemblies of two and three particles as the streamer tip approaches the top particle. The two particles are spaced far enough apart so that the polarization of the top particle produced by the streamer does not significantly affect the lower particle. The three particles are spaced closely enough so that the streamer induced polarization of the top particle couples with and polarizes the lower particles. This has an important implication on the streamer-particle interaction.

Plasma properties during the interaction of a streamer with three equidistant particles $80 \mu \mathrm{m}$ in radius and with $\varepsilon / \varepsilon_{0}=5$ are shown in figure 12. The initial stage of interaction with the top particle is similar to that for a single isolated particle. As with the isolated particle, following the intersection of the streamer with the top of the particle, a second streamer is launched from the bottom of the first particle. Polarization of the particle produces a larger electric field below the particle which, when seeded by photoionization, produces an electron temperature exceeding $7 \mathrm{eV}$ and an avalanche with electron source, $S_{\mathrm{e}}$, exceeding $10^{25} \mathrm{~cm}^{-3} \mathrm{~s}^{-1}$. In contrast, the electron temperature near the particle's equator is around $3 \mathrm{eV}$ leading to smaller values of $S_{\mathrm{e}}$.

The second particle is within the polarization field of the first. As a consequence, as the second streamer is launched from below the first particle and begins to create a conductive channel; the electric field in front of the second streamer is compressed between the tip of the streamer and the surface of the second particle. This serves to increase 

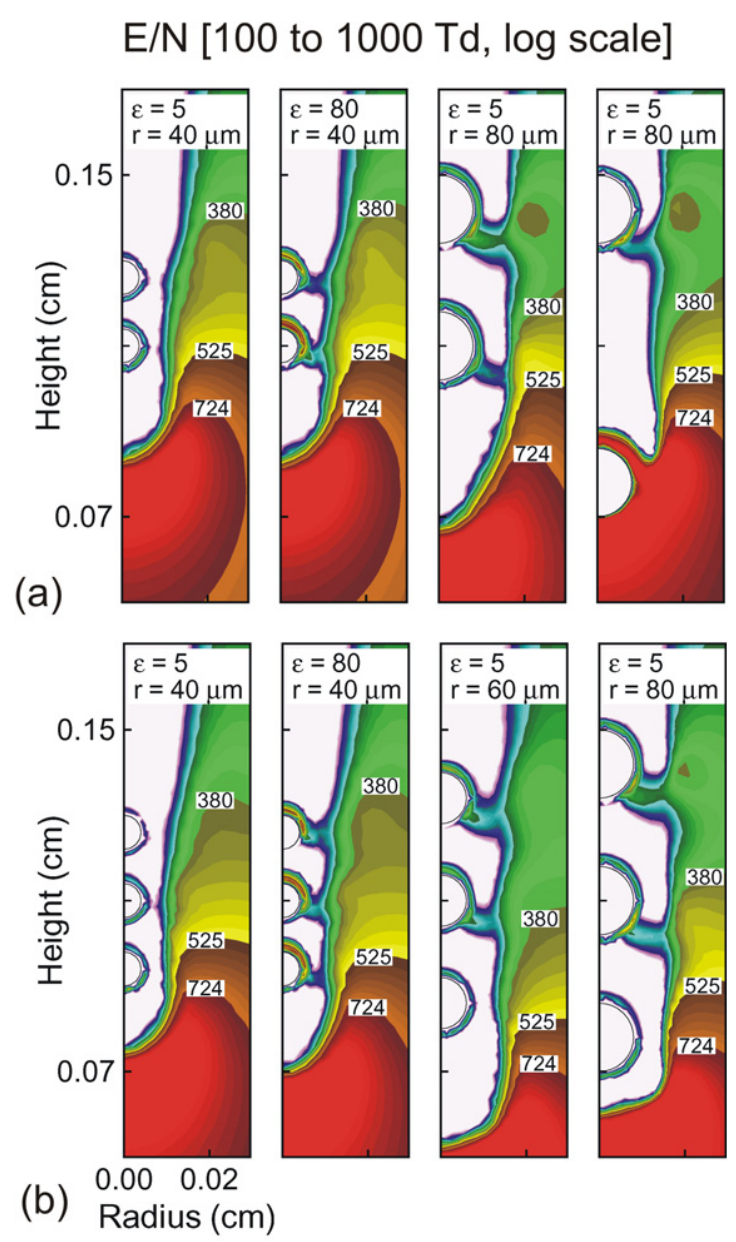

MIN

Figure 13. Reduced electric fields around particles of varying size, spacing and permittivity. $E / N$ around (a) two particles at $t=4.5 \mathrm{~ns}$ and $(b)$ three particles at $t=4.65 \mathrm{~ns}$. Contour labels are in townsend. Small particles or particles with low dielectric constant are enveloped by the streamer plasma. Particles with high dielectric constant reinitiate the streamer. The process also depends on the distance between the particles.

the polarization field at the top of the second particle. This results in rapid ionization, avalanche between the top two particles and charging of the top of the second particle. The polarization field at the bottom of the second particle is seeded by photoionization, and a third streamer is launched from its lower surface. This process is repeated on the third particle resulting in launching of a fourth streamer from its bottom side.

This process of successively launching streamers from the bottom of particles is very much like a relay in which the streamer is handed off between particles. There is electric field enhancement at the upper pole of the particle, particle charging, electric field enhancement at the lower pole and launching of the daughter streamer. This process is repetitive for particles that are of the same size and evenly spaced as in this example. Randomly spaced and sized particles display more chaotic behaviour.

As is the case with an isolated particle, the particles' sizes and permittivities, as well as their inter-particle spacing, determine the character of streamer propagation through

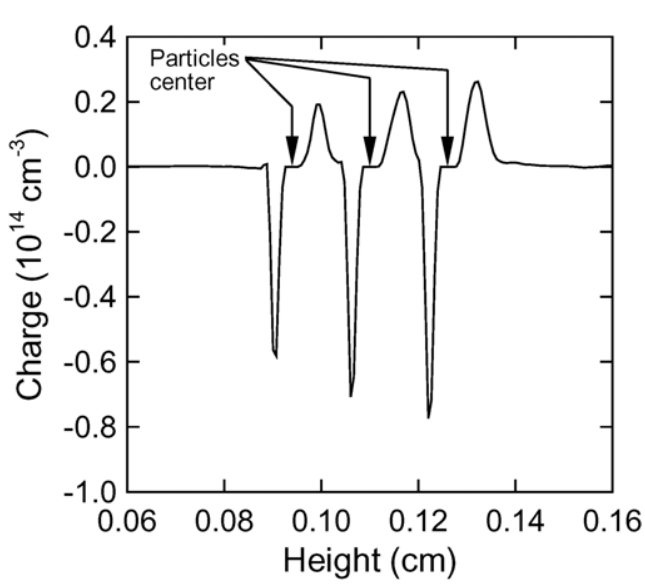

Figure 14. Charge density along the axis in the gas phase and accumulated on the surface of three equidistant $40 \mu \mathrm{m}$ radius particles with $\varepsilon=5$. Propagation is from right to left. Typically, the first particle in the streamer path accumulates the largest positive and negative charge.

multiple particles. Some of these trends are shown in figure 13. Small particles $(<60 \mu \mathrm{m})$ with low dielectric constant $\left(\varepsilon / \varepsilon_{0}<\right.$ 20-30) are enveloped by the streamer plasma (indicated by the low values of $E / N$ ) while large particles or particles with high dielectric constant stall and reinitiate the streamer. The distance between the particles is also important as closely spaced particles more effectively induce high electric fields on adjacent particles, thereby producing a more rapid avalanche.

Although streamers can be efficiently handed off from particle to particle, the streamers do tend to weaken as the streamer stalls and is reinitiated. This is, in part, a consequence of charge being removed from the streamer and being deposited on the particles. Typically, the first particle in the path of a streamer accumulates both the largest positive and negative charge as shown in figure 14.

Propagation speeds of streamers intersecting with single and multiple particles typically increase with the size of the particle as long as the particle radius is not significantly larger than the streamer radius. The larger local electric fields produced by polarization of the particle accelerate the avalanche process. When the particle is large in comparison with the streamer radius, the streamer speed may decrease. The streamer stalls while charging the particle and the particle shadows photoionization from seeding the second streamer from the bottom of the particle. In this case the particle acts as an obstacle where the streamer spends additional time to charge, envelope the particle and reinitiate a new streamer.

\section{Concluding remarks}

Results from a computational investigation of the dynamics of positive corona streamers in humid air intersecting with dielectric particles were discussed. We found that the propagation of these streamers can be significantly perturbed by particles due, in part, to the polarization of the particles that produces larger electric fields at their poles. These large electric fields facilitate larger rates of ionization. Small particles $(<$ tens of micrometres) of moderate permittivity $\left(\varepsilon / \varepsilon_{0}<10\right)$ are typically enveloped by the streamer and do not significantly perturb streamer propagation. Larger particles can intercept the 
streamer while charging and, if large enough to shadow photoionization from providing seed electrons below the particle, stall propagation of the streamer. As the plasma propagates around and envelopes the particle, and photoionizing radiation illuminates the bottom of the particle where the polarization field is large, a second streamer can be launched. Synergistic effects of closely spaced particles whose polarization fields overlap can produce a series of stalling and re-launching of streamers. Under these conditions, the speed of the streamer may increase in qualitative agreement with experiments [9].

\section{Acknowledgment}

This work was supported by the National Science Foundation (CTS-0520368).

\section{References}

[1] van Veldhuizen E M (ed) 1999 Electrical Discharges for Environmental Purposes: Fundamentals and Applications (New York: Nova Science).

[2] Reist P C 1993 Aerosol Science and Technology (New York: McGraw-Hill)

[3] Bouchoule A (ed) 1999 Dusty Plasmas, Physics, Chemistry and Technological Impacts in Plasma Processing (New York: Wiley)
[4] Starikovskaia S M, Kukaev E N, Kuksin A Yu, Nudnova M M and Starikovskii A Yu 2004 Combust. Flame 139177

[5] Wang F, Liu J B, Sinibaldi J, Brophy C, Kuthi A, Jiang Cl, Ronney P and Gundersen M A 2005 Trans. Plasma Sci. 33844

[6] Yurteri C U, Mazumder M K, Grable N, Ahuja G, Trigwell S, Biris A S, Sharma R and Sims R A 2002 Particulate Sci. Technol. 2059

[7] Babaeva N Yu and Naidis G V 1996 J. Phys. D: Appl. Phys. 292423

[8] Kulikovsky A A 1997 J. Phys. D: Appl. Phys. 301515

[9] Tardiveau P and Marode E 2003 J. Phys. D: Appl. Phys. 361204

[10] Timatkov V V, Pietsch G J, Saveliev A B, Sokolova M V and Temnikov A G 2005 J. Phys. D: Appl. Phys. 38877

[11] Briels T M, Veldhuizen E M and Ebert U 2005 IEEE Trans. Plasma Sci. 33264

[12] Kushner M J 2005 J. Phys. D: Appl. Phys. 381633

[13] Scharfetter D L and Gummel H K 1969 IEEE Trans. Electron. Dev. ED-16 64

[14] Dorai R and Kushner M J 2003 J. Phys. D: Appl. Phys. 36666

[15] van Veldhuizen E M and Rutgers W R 2002 J. Phys. D: Appl. Phys. 352169

[16] Cheng D K 1992 Field and Wave Electromagnetics (Reading: Addison-Wesley).

[17] Babaeva N Yu, Lee J K and Kim H C 2004 Plasma Sources Sci. Technol. 13127 